\title{
Thyroid-stimulating hormone is associated with trabecular bone score and 5-year incident fracture risk in euthyroid postmenopausal women: the OsteoLaus cohort
}

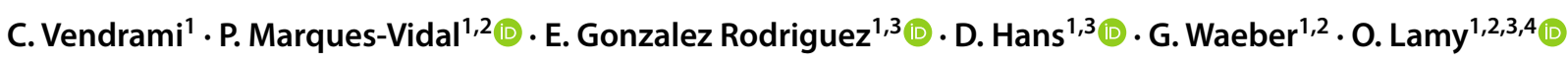

Received: 23 April 2021 / Accepted: 21 July 2021 / Published online: 19 August 2021

(c) The Author(s) 2021

\begin{abstract}
Summary Thyroid-stimulating hormone (TSH) excess or deficiency influences bone density and fracture risk. Nevertheless, does TSH in the reference range influence bone health? In euthyroid postmenopausal women, TSH levels in the reference range were positively associated with trabecular bone score and negatively with incident fractures, without affecting BMD. Purpose Subclinical hyperthyroidism is associated with low bone mineral density (BMD) and increased fracture risk. In healthy postmenopausal women, association between thyroid-stimulating hormone (TSH) in the normal range and BMD is contradictory. Trabecular bone score (TBS), an index of bone micro-architecture, is often decreased in secondary osteoporosis (OP). The aim was to determine the association between thyroid hormones (TSH, fT4) and BMD, TBS, and the incident 5 -year OP fractures, in euthyroid post-menopausal women.

Methods We assessed 1475 women of the CoLaus/OsteoLaus cohort. We evaluated BMD at lumbar spine, femoral neck and total hip, lumbar spine TBS, and vertebral fracture with DXA. Incident major OP fractures were evaluated 5 years later by questionnaire and DXA. Women with anti-osteoporotic, antidiabetic, thyroid-modifying, hormone replacement, or systemic corticoid treatment were excluded.

Results Five hundred thirty-three women (age $68.4 \pm 7.3$ years, BMI $25.9 \pm 4.6 \mathrm{~kg} / \mathrm{m}^{2}$, TSH $2.03 \pm 0.87 \mathrm{mU} / 1$, fT4 $15.51 \pm 1.85 \mathrm{pmol} / \mathrm{l})$ met the inclusion criteria. There was no significant association between TSH or fT4 and BMD measures at any site. A positive association was found between TSH and TBS $(\beta=0.138, p<0.01)$, even after adjusting for age, BMI, and duration of menopause $(\beta=0.086, p<0.05)$. After a 5 -year follow-up, women with incident major OP fractures had lower TSH levels $(1.77 \pm 0.13$ vs. $2.05 \pm 0.04 \mathrm{mU} / \mathrm{l}, p<0.05)$ than women without fractures, while no difference was found for fT4. Conclusion In euthyroid postmenopausal women, TSH levels were positively associated with TBS and negatively with incident fractures, without affecting BMD. Further studies are needed to evaluate the influence of thyroid hormones on TBS.
\end{abstract}

Keywords Thyroid hormones · Fractures · Osteoporosis · Bone mineral density $\cdot$ Trabecular bone score

\section{Introduction}

O. Lamy

olivier.lamy@chuv.ch

1 University of Lausanne, UNIL, CHUV, Lausanne, Switzerland

2 Internal Medicine Unit, Internal Medicine Department, CHUV, Lausanne, Switzerland

3 Center of Bone Diseases, Rheumatology Unit, Bone and Joint Department, CHUV, Lausanne, Switzerland

4 Department of Medicine, Rue du Bugnon 46, 1011 Lausanne, Switzerland
Osteoporosis is characterized by a deterioration of bone microarchitecture and a loss of bone mineral density (BMD), leading to an increase of fracture risk. Worldwide, about $50 \%$ of women and $20 \%$ of men over 50 years will be affected by an osteoporotic fracture [1]. However, half of fractures occurs in women and men who do not have a low BMD [2-4]. The FRAX® tool includes clinical data to predict the 10-year fracture risk [5], while the trabecular bone score (TBS) is a clinical tool to quantify the texture variations extracted from the lumbar spine dual-energy X-ray absorptiometry (DXA) [6]. TBS provides information on bone micro-architecture that is complementary to BMD and 
FRAX®, and independently and significantly predicts fracture [7].TBS is slightly associated with BMD but not with degenerative disease or osteoarthritis [8]. TBS also gives an accurate result of bone texture in patients aged over 60 years, where the lumbar spine BMD tend to be overestimated [9].

Thyroid function is one of the multiple risk factors for osteoporosis. Thyroid dysfunction is common in the general population, especially in postmenopausal women, and increases with age. Hypothyroidism is present in $4.6 \%$ of the US population and hyperthyroidism in $1.3 \%$ [10]. The prevalence of subclinical hypothyroidism (defined by an elevated thyroid-stimulating hormone (TSH) level with free thyroxine (fT4) within the reference range) in the adult population ranges from 4 to $20 \%$, depending on age, sex, body mass index (BMI), race, dietary iodine intake, and threshold serum TSH levels used [11]. Hypothyroidism results in low bone turnover with a decrease of bone formation and resorption, but the consequences on BMD in adults are debated. In a meta-analysis including 313,557 individuals, subclinical hypothyroidism was associated with an increased femoral neck BMD in women [12], while in another meta-analysis including 762,401 person-years follow-up, there was no association between subclinical hypothyroidism and fracture [13]. Hyperthyroidism is a well-known cause of excessive bone remodeling, associated with accelerated bone loss leading to osteoporosis. The prevalence of subclinical hyperthyroidism varies between 1 and 9.8\% [11]. Compared to euthyroidism, subclinical hyperthyroidism is associated with an increased bone loss at the femoral neck [14]. The negative effect on lumbar spine BMD and total hip BMD was only present at the lowest TSH values. High thyroid function, even at high-normal levels, increases fracture risk [13, 15].

In euthyroid adults, the combination of a TSH in the lower normal reference range and a fT4 in the higher normal reference range are associated with an increased risk of hip fracture [15]. Published studies in euthyroid individuals suggest that fT4 influences BMD, but only in case of high value and only on some measurement sites. There is some controversy as to whether alterations in $\mathrm{TSH}$, or peripheral hormones, or both, are necessary to affect bone homeostasis. TBS is often decreased in secondary osteoporosis [16]. To date, only one study has characterized the relationship between thyroid function and TBS in euthyroid men and postmenopausal women [17]. Higher fT4 levels within the normal reference range were associated with deterioration of TBS in healthy euthyroid postmenopausal women, without affecting the BMD. In the TRUST study, a one-year levothyroxine treatment had no effect on TBS in older adults with subclinical hypothyroidism [18].

Hence, this study aimed to determine the association between TSH and fT4 in the normal range and BMD, TBS, and incident fractures at 5 years in a cohort of Caucasian postmenopausal women: the OsteoLaus study.

\section{Materials and methods}

\section{Settings}

OsteoLaus is a sub-study of the CoLaus|PsyColaus study, an ongoing prospective study aiming to assess the determinants of cardiovascular and psychiatric diseases using a population-based sample $[19,20]$. Information on the methodology of the CoLauslPsyCoLaus study can be obtained at www.colaus-psycolaus.ch. Briefly, the CoLaus study was initiated in 2003; it included 6733 men and women aged 35 to 75 years living in the city of Lausanne, Switzerland. Follow-up has been done every 5 years, first in 2009, second in 2014, and third in 2018 .

The goal of OsteoLaus is to obtain more precise fracture risk models and to evaluate the link between cardiovascular diseases and osteoporosis [21]. Between September 2009 and September 2012, all women aged between 50 and 80 years from the CoLausIPsyColaus study were invited to participate in OsteoLaus. Of the initial 1704 women invited, 1500 (88\%) accepted, and 1475 were included; 98.4\% were Caucasian. Follow-up of OsteoLaus has been done every 2.5 years, first in 2012, second in 2015, and third in 2018 .

\section{Participants}

The study was retrospective. Participants in the 5-year follow-up of OsteoLaus were included $(n=1212)$. Blood TSH and free $\mathrm{T}_{4}$ were assessed at the first CoLaus follow-up, corresponding to the baseline OsteoLaus visit. Participants were sequentially excluded if they 1) had any anti-osteoporotic, antidiabetic, thyroid-modifying, hormone replacement or systemic corticoid treatment; 2) had no TSH or fT4 data, or no covariates; 3) presented with TSH or fT4 levels outside the normal reference values, and 4) had no densitometry or TBS data.

The primary endpoint of this study was to analyze the relation between $\mathrm{fT} 4$ and TSH in the normal range and bone measurements parameters (lumbar spine BMD, femoral neck BMD, total hip BMD, and TBS). The primary endpoint included an exploratory comparison of two subgroups divided by the medians of TSH and fT4: high fT4/low TSH and low fT4/high TSH. The secondary endpoint was to analyze the association between fT4 and TSH measured at baseline and the incidence of major osteoporotic fracture 5 -year later.

\section{Bone parameters}

BMD, TBS, and prevalence of fractures were assessed at baseline OsteoLaus visit using the following: (1) a 
questionnaire on potential clinical risk factors for fracture/ osteoporosis, and on conditions affecting bone metabolism; (2) a spine (L1 to L4) and femur dual DXA scan using the Discovery A System (Hologic, Waltham, MA, USA); (3) a blind central processing of TBS (TBS iNsight ${ }^{\circledR} \mathrm{v} 3.03$, Medimaps, Mérignac, France) based on the previously acquired antero-posterior spine DXA scan; (4) a vertebral fracture assessment (VFA) by two experimented clinicians using the semiquantitative approach of HK Genant [22]. Spine and femur DXA were analyzed according to the International Society for Clinical Densitometry (ISCD) recommendations [23]. The current TBS software is based on an algorithm accounting for BMI. We also used the research TBS algorithm which takes into account the soft tissue thickness TBS TTH [24]. Major osteoporotic fractures included vertebrae (clinical or radiologic grade 2/3 on VFA), hip, pelvis, humerus and radius, occurring spontaneously or after falling from the patient's own height. Incidence of major osteoporotic fractures was assessed similarly during the second OsteoLaus follow-up after 5 years.

\section{Blood measurements}

Assays were performed by the CHUV Clinical Laboratory on fresh blood samples within $2 \mathrm{~h}$ after collection. TSH and $\mathrm{fT}_{4}$ were assessed by chemiluminescence (ECLIA) on a Cobas e 602 device (Roche diagnostics GmbH, Mannheim, Germany) with intra-batch coefficients of variation ranging between 1.1 and 3.0\% for TSH and between 2.7 and 5\% for $\mathrm{fT}_{4}$. Technical documentation (in French) can be provided upon request. Reference ranges are 0.27 to $4.20 \mathrm{mU} / \mathrm{L}$ for TSH and 12 to $22 \mathrm{pm} / \mathrm{L}$ for $\mathrm{fT}_{4}$ [25]. Serum and urinary creatinine were measured by the Jaffe kinetic compensated method (2.9-0.7\%). Estimated glomerular filtration rate (eGFR) was computed using the MDRD equation.

\section{Covariates}

Height and weight were measured with the participant barefoot and in minimum clothing, using a portable stadiometer/electronic scale (Seca version 216, Seca, Chino, CA, USA) with a precision of $0.1 \mathrm{~cm}$ and $0.1 \mathrm{~kg}$, respectively. BMI was calculated by dividing the individual's weight by height squared $\left(\mathrm{kg} / \mathrm{m}^{2}\right)$. Age, history of disease, smoking status, alcohol consumption, and menopausal duration were assessed by questionnaire. Menopause duration was categorized into $\leq$ and $>10$ years.

\section{Statistical methods}

Statistical analysis was conducted using Stata v.16 (Stata Corp, College Station, TX, USA). Prevalence levels and corresponding 95\% confidence intervals for deteriorated TBS (values $<1.23$ ) or BMD (values $<-2.5 \mathrm{SD}$ ) were computed using the exact binomial procedure. Bivariate associations were conducted using Spearman correlations; multivariate associations were conducted using linear regression and the results were expressed as beta standardized coefficients. Multivariate linear regression used bone measures as the dependent variable and thyroid hormones as the independent variable, with adjustment for age (continuous), BMI (continuous), and duration of menopause (categorical). Statistical significance was considered for a two-sided test with $p<0.05$. As previous studies suggested that low-normal TSH (i.e., $<1 \mathrm{mU} / \mathrm{l}$ in the normal range) may favor skeletal fragility and higher risk of fractures, a sensitivity analysis was conducted by comparing bone parameters according to three groups of TSH $(<1,1-2.5$, and $>2.5 \mathrm{mU} / \mathrm{l})$. Further comparisons between participants with TSH levels below or above $1 \mathrm{mU} / \mathrm{L}$ were conducted among participants who experienced fragility fractures after 5-year follow-up. As bone parameters at baseline were strongly correlated (Spearman $r$ ranging from 0.366 to 0.851 , all $p<0.0001$ ), several multivariable analyses adjusting for other covariates and for one parameter at the time were conducted.

\section{Ethics committee}

The CoLaus and OsteoLaus studies were approved by the Institutional Ethics Committee of the University of Lausanne, which afterwards became the Ethics Commission of Canton Vaud (Commission cantonale d'éthique de la recherche sur l'être humain. Available from: http://www. cer-vd.ch). The studies were performed in agreement with the Helsinki Declaration and its former amendments, and in accordance with the applicable Swiss legislation. All participants gave their written informed consent before entering the study.

\section{Results}

\section{Participants' selection and characteristics}

The selection procedure of the participants is shown in Fig. 1. Of the initial 1212 participants, $533(45.6 \%)$ had normal values for TSH and fT4 (Supplementary Material, Fig. 1). The clinical characteristics of excluded and included participants are provided in Table 1. There were no significant differences between the two groups except for age, with the excluded participants being 1.5 years older. No included participant has further confounders such as renal failure (GFR $\left.<15 \mathrm{ml} / \mathrm{min} / 1.73 \mathrm{~m}^{2}\right)$, anticancer drugs, genetic bone disease or malformation, inflammatory bowel disease, gastrectomy, anorexia, neoplasm 
Fig. 1 Flowchart with exclusion criteria

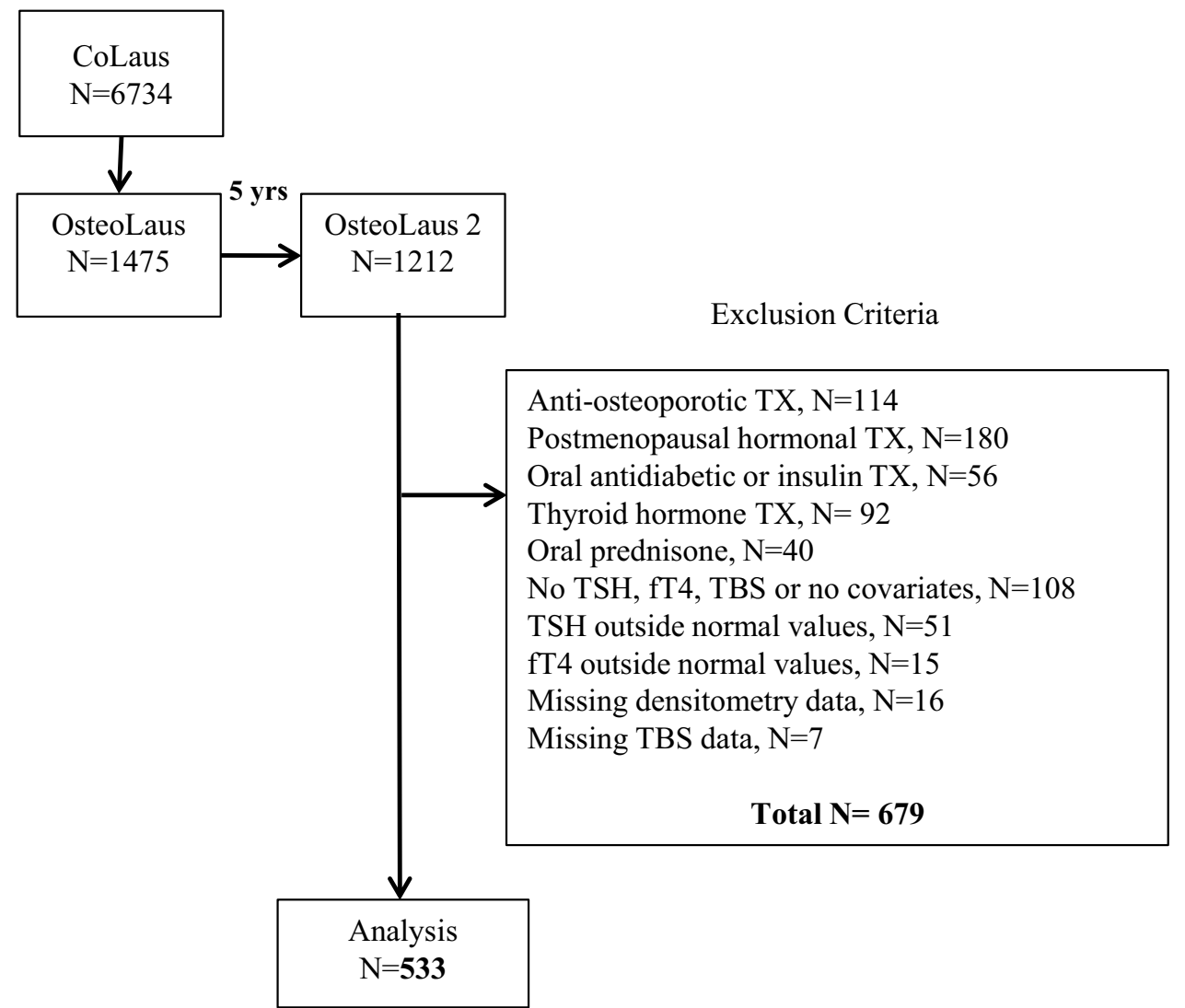

\begin{tabular}{llllc}
\hline & Baseline & Included & Excluded & $p$-value \\
\hline$n$ & 1212 & 533 & 679 & - \\
Age $($ year) & $69.27 \pm 7.38$ & $68.44 \pm 7.32$ & $69.93 \pm 7.37$ & 0.001 \\
BMI $\left(\mathrm{kg} / \mathrm{m}^{2}\right)$ & $26.07 \pm 4.72$ & $25.88 \pm 4.55$ & $26.22 \pm 4.86$ & 0.221 \\
Duration of menopause (years) & $20.09 \pm 9.50$ & $19.01 \pm 9.34$ & $21.20 \pm 9.53$ & 0.000 \\
eGFR $\left(\mathrm{ml} / \mathrm{min} / 1.73 \mathrm{~m}^{2}\right)$ & $71.88 \pm 13.66$ & $72.24 \pm 12.90$ & $71.59 \pm 14.25$ & 0.410 \\
Current smoker & $15.19 \%$ & $14.82 \%$ & $15.49 \%$ & 0.749 \\
Femoral neck BMD $T$-score & $-1.225 \pm 0.874$ & $-1.285 \pm 0.820$ & $-1.177 \pm 0.914$ & 0.034 \\
Total hip BMD $T$-score & $-0.876 \pm 1.014$ & $-0.939 \pm 0.970$ & $-0.824 \pm 1.046$ & 0.053 \\
Lumbar spine BMD $T$-score & $-0.640 \pm 1.640$ & $-0.747 \pm 1.572$ & $-0.553 \pm 1.690$ & 0.043 \\
TBS & $1.314 \pm 0.095$ & $1.315 \pm 0.094$ & $1.313 \pm 0.096$ & 0.705 \\
Fractures at 2.5 years & $6.19 \%(75)$ & $5.63 \%(30)$ & $6.63 \%(45)$ & 0.474 \\
Fractures at 5 years & $9.65 \%(117)$ & $8.26 \%(44)$ & $10.75 \%(73)$ & 0.144 \\
\hline
\end{tabular}

Fractures accounted are the major osteoporotic fractures: non traumatic vertebral, hip, humerus, and forearm or other secondary osteoporosis. The prevalence of $\mathrm{BMD}<-2.5 \mathrm{DS}$ was $10.9 \%$ (95\% CI 8.4-13.8) at lumbar spine, 3.9\% (95\% CI 2.5-6.0) at total hip, and 5.3\% (95\% CI 3.5-7.5) at femoral neck. Deteriorated TBS was present in $18.4 \%$ (95\% CI $15.2-21.9$ ).

\section{TSH, fT4, and bone parameters: main analysis}

There was no association between fT4 and lumbar spine or hip BMD (Table 2 and Fig. 2). As the results for TBS and TBS TTH (algorithm used for research) were the same, we 
Table 2 Association between TSH and fT4 and BMD and TBS

\begin{tabular}{lccllll}
\hline & Spearman & $p$-value & Beta, model 1 & $p$-value & Beta, model 2 & $p$-value \\
\hline TSH & & & & & & \\
Femoral neck BMD T-score & 0.040 & 0.355 & 0.048 & 0.271 & 0.023 & 0.576 \\
Total hip BMD T-score & 0.058 & 0.181 & 0.064 & 0.142 & 0.045 & 0.256 \\
Lumbar spine BMD T-score & 0.015 & 0.727 & 0.013 & 0.771 & 0.010 & 0.805 \\
TBS & $\mathbf{0 . 1 3 2}$ & $\mathbf{0 . 0 0 2}$ & $\mathbf{0 . 1 3 8}$ & $\mathbf{0 . 0 0 1}$ & $\mathbf{0 . 0 8 7}$ & $\mathbf{0 . 0 3 1}$ \\
fT4 & & & & & & \\
Femoral neck BMD T-score & -0.010 & 0.821 & 0.013 & 0.758 & -0.014 & 0.732 \\
Total hip BMD T-score & 0.002 & 0.966 & 0.014 & 0.755 & -0.017 & 0.669 \\
Lumbar spine BMD T-score & 0.020 & 0.654 & 0.016 & 0.706 & 0.006 & 0.518 \\
TBS & $\mathbf{0 . 0 9 4}$ & $\mathbf{0 . 0 2 6}$ & $\mathbf{0 . 1 0 5}$ & $\mathbf{0 . 0 1 5}$ & 0.066 & 0.101 \\
\hline
\end{tabular}

Results are expressed as Spearman rank correlation or beta coefficients obtained from linear regression. Statistical analysis performed using Spearman rank correlation or linear regression. Model 1: unadjusted; model 2: adjusted for age (continuous), body mass index (continuous), and duration of menopause ( $\leq$ or $>10$ years)
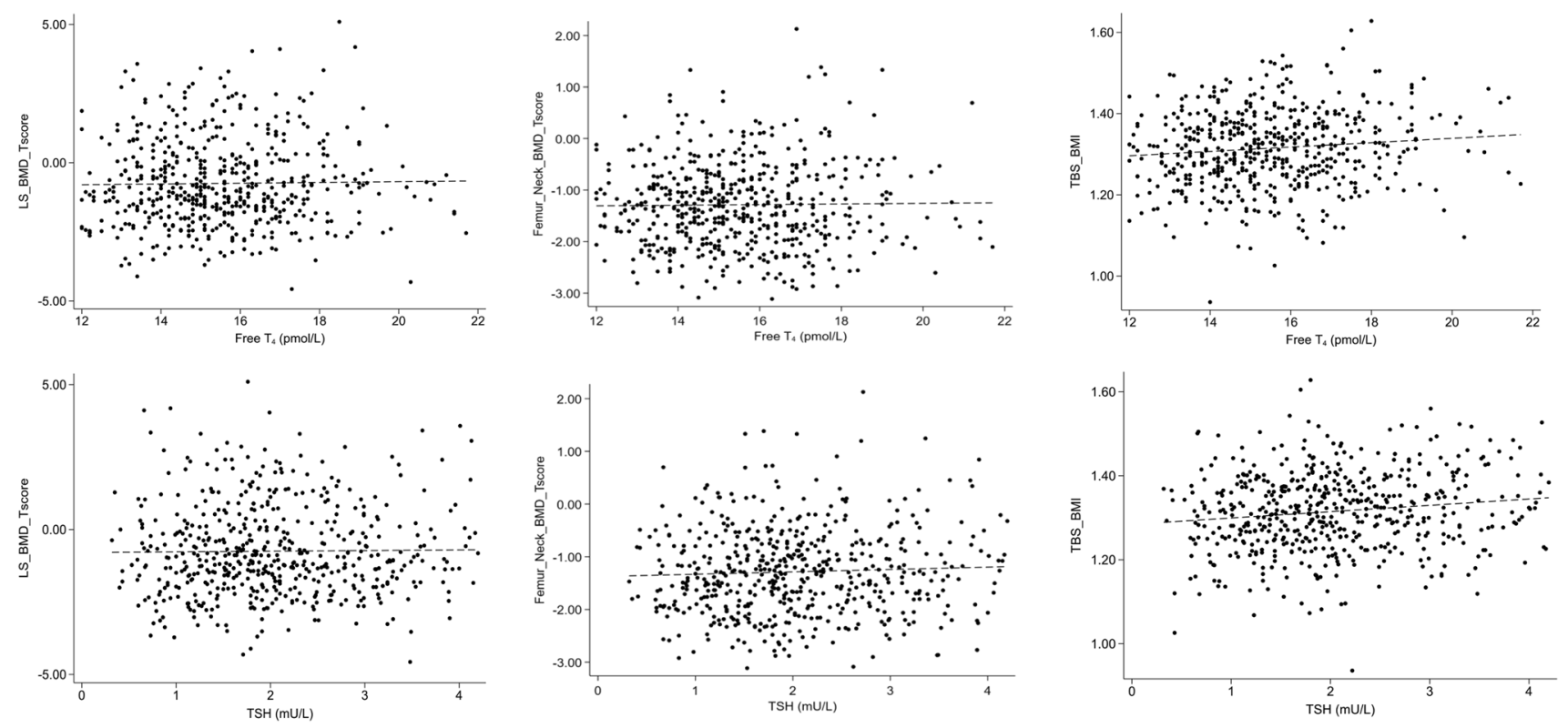

Fig. 2 Association between fT4 and TSH and lumbar spine (LS) BMD $T$-score, femoral neck BMD $T$-score, and TBS with regression line

give only the results for the TBS used in clinical routine. fT4 was positively associated with TBS $(\beta=0.105, p=0.015)$ in the unadjusted model but disappeared after adjustment. TSH was positively associated with TBS $(\beta=0.138, p<0.01)$ in the unadjusted model, and remained significant after adjustment for age, BMI and duration of menopause $(\beta=0.087$, $p=0.031)$.

\section{TSH, fT4, and bone parameters: exploratory analysis}

The purpose of this exploratory analysis was to detect a difference within the extremes of the normal fT4 and TSH values. We compared two subgroups divided by the medians of TSH and fT4: high fT4/low TSH $(n=137)$ and low
fT4/high TSH $(n=136)$. Neither BMD values at the three measurement sites, nor TBS values differed between the two groups in the bivariate and multivariable analyses (Table 3). In the Supplementary Table 1, we analyzed TBS, BMD and fractures in subjects stratified in three groups TSH categories $(\mathrm{TSH}<1 \mathrm{mU} / 1$, TSH $1-2.5 \mathrm{mU} / 1$ and $\mathrm{TSH}>2.5 \mathrm{mUl}$ ). The conclusions do not change relative to the comparison between high fT4/low TSH and low fT4/high TSH groups.

\section{Incident fractures}

After 2.5 and 5 years, 30 and 44 participants, respectively, had a major incident osteoporotic fracture. Among these 44 participants, six had vertebral fracture and nine had 
Table 3 Association between BMD and TBS with categories of fT4 and TSH

\begin{tabular}{llll}
\hline & High fT4, low TSH & Low fT4, high TSH & $p$-value \\
\hline$n$ & 137 & 136 & \\
Femoral neck & $T$-score & & \\
Model 1 & $-1.28 \pm 0.88$ & $-1.23 \pm 0.77$ & 0.637 \\
Model 2 & $-1.3 \pm 0.07$ & $-1.22 \pm 0.07$ & 0.357 \\
Total hip $T$-score & & \\
Model 1 & $-0.92 \pm 1.04$ & $-0.94 \pm 0.89$ & 0.888 \\
Model 2 & $-0.95 \pm 0.07$ & $-0.90 \pm 0.07$ & 0.631 \\
Lumbar spine & $T$-score & & \\
Model 1 & $-0.71 \pm 1.64$ & $-0.77 \pm 1.46$ & 0.752 \\
Model 2 & $-0.74 \pm 0.13$ & $-0.74 \pm 0.13$ & 0.988 \\
TBS & & & \\
Model 1 & $1.31 \pm 0.1$ & $1.31 \pm 0.09$ & 0.837 \\
Model 2 & $1.31 \pm 0.01$ & $1.32 \pm 0.01$ & 0.522 \\
\hline
\end{tabular}

Results are expressed as average \pm standard deviation or as multivariable-adjusted average \pm standard error. Model 1: unadjusted; model 2: adjusted for age (continuous), body mass index (continuous), and duration of menopause ( $\leq$ or $>10$ years)

Table 4 Association between TSH and fT4 and incident major osteoporotic fractures at 2.5 and 5 years

\begin{tabular}{cllllll}
\hline & $n$ & \multicolumn{2}{l}{ TSH $(\mathrm{mU} / \mathrm{L})$} & & \multicolumn{2}{l}{$\mathrm{fT}$ 4 $(\mathrm{pmol} / \mathrm{L})$} \\
\cline { 7 - 7 } \cline { 6 - 7 } & & Model 1 & Model 2 & & Model 1 & Model 2 \\
\hline 2.5 years & & 0.074 & 0.134 & & 0.494 & 0.613 \\
No & 503 & $2.05 \pm 0.86$ & $2.04 \pm 0.04$ & & $15.5 \pm 1.9$ & $15.5 \pm 0.1$ \\
Yes & 30 & $1.75 \pm 0.90$ & $1.80 \pm 0.16$ & & $15.3 \pm 1.6$ & $15.3 \pm 0.3$ \\
5 years & & 0.013 & 0.038 & & 0.975 & 0.746 \\
No & 489 & $2.06 \pm 0.87$ & $2.05 \pm 0.04$ & & $15.5 \pm 1.9$ & $15.5 \pm 0.1$ \\
Yes & 44 & $1.72 \pm 0.83$ & $1.77 \pm 0.13$ & & $15.5 \pm 1.8$ & $15.6 \pm 0.3$ \\
\hline
\end{tabular}

Results are expressed as average \pm standard deviation or as multivariable-adjusted average \pm standard error. Model 1: unadjusted; model 2: adjusted for age (continuous), body mass index (continuous), and duration of menopause $(\leq$ or $>10$ years $)$

two major osteoporotic fractures. After a 5-year follow-up, participants with major osteoporotic fractures had lower TSH levels than participants without fracture in the bivariate analysis $(1.77 \pm 0.13$ vs. $2.05 \pm 0.04 \mathrm{mU} / 1, p=0.038$, Table 4); this association remained after adjusting for age, BMI, and menopause duration. No association between TSH and incident fractures at 2.5 years was detected. There was no association between fT4 and the incident fracture after 2.5 or 5 years. Among participants who experienced fragility fractures after 5-year follow-up, we provided in Supplementary Table 2 the distribution of TBS and BMD in those with TSH below $1 \mathrm{mU} / \mathrm{L}$ and in those with TSH $>1 \mathrm{mU} / \mathrm{l}$. We chose the 5-year follow-up to have the maximum number of participants and thus the highest statistical power possible. There was no difference in BMD or TBS values. There were strong correlations between all baseline variables (Spearman $r$ ranging from 0.366 to 0.851 , all $p<0.0001$ ). Hence, to avoid collinearity, it was decided to adjust on a single variable, and several models were applied, one for each variable. The results are provided in Supplementary Table 3. The results confirm those indicated in Table 4, i.e., the probable existence of an association between TSH levels and incident fractures.

\section{Discussion}

In this cross-sectional study of postmenopausal euthyroid women, there was a positive association between TSH and TBS. In the longitudinal analysis, there was an inverse association between TSH and incident major osteoporotic fractures at 5 years.

\section{Association between fT4 or TSH and BMD or TBS in euthyroid postmenopausal women}

We found a positive association between TSH and TBS in postmenopausal women. The association between $\mathrm{fT} 4$ and TBS on bivariate analysis disappeared on multivariable analysis. The finding of an association between TSH and TBS, but not fT4 and TBS, is probably due to the higher sensitivity of TSH as a biomarker and the insufficient power to identify an association with fT4. In a Korean study including 648 postmenopausal women, fT4 was negatively associated with TBS, while no association was found between TSH and TBS or BMD [17]. Although both studies go in the same direction (a lower thyroid function is associated with higher TBS), the difference can be due to differences in participants: ethnicity, younger age, lower BMI, the use of a different DXA facility (GE Lunar Prodigy vs. Hologic) and the exclusion criteria. Two studies of thyroid cancer patients receiving exogenous T4 treatment to suppress TSH found the same trends. In 273 postmenopausal women with differentiated thyroid carcinoma, duration of TSH suppression was negatively correlated with lumbar spine TBS levels, but not with BMD [26]. Similar results were found in 84 Spanish postmenopausal women (but not in the 61 premenopausal women) treated with thyroid suppression therapy for differentiated thyroid carcinoma [27]. The authors concluded that TBS should be considered in the evaluation of these postmenopausal women for fracture risk evaluation. This association with altered TBS, but not with altered BMD, has been described in other causes of secondary osteoporosis (type 2 diabetes mellitus, chronic kidney disease glucocorticoids, HIV) [16].

We and the previously cited studies [17, 26, 27] found no association between TSH or fT4 and BMD, contrary to other studies [12, 28-31]. Mazziotti G et al. found an 
association between low-normal TSH values and high prevalence of vertebral fractures in women, independently of thyroid hormones, age and BMD [29]. In 1278, European postmenopausal women ( $68 \pm 7$ years), higher $\mathrm{fT}_{4}$ levels within the normal range were associated with lower hip BMD [30]. There was no association between lumbar spine BMD and fT4. Therefore, it is possible that participants with hyper- or hypothyroidism might have been included $\left(2.5^{\text {th }}\right.$ and $97.5^{\text {th }}$ percentiles values for fT4 and TSH). In a sample of 1426 peri-menopausal Dutch women, fT4 levels in the highest quartile within the normal range, but not low serum TSH, were independently related to decreased BMD at lumbar spine [31]. In a metaanalysis including 313,557 individuals (mean age from 51 to 85 years), in women, subclinical hyperthyroidism was associated with lower distal forearm BMD, whereas subclinical hypothyroidism (high TSH with normal fT4) was associated with higher femoral neck BMD [12]. The differences observed at lumbar spine between both studies may be related to the age of the participants. As TBS is not associated with degenerative disease or osteoarthritis, it gives an accurate result in patients over 60 year, where the lumbar spine BMD is overestimated [9].

The protective effect of TSH on bone has been demonstrated in in vitro studies by uncoupling bone resorption and formation, leading to an increase in bone mass [32-34]. Only one in vitro study suggested that the effect of the thyrotropic axis on bone is due to the thyroid hormone effects instead of the direct effects of TSH itself [35]. A study in 10 thyroidectomized postmenopausal women treated with a recombinant TSH injection showed an increase in bone formation markers; this result was absent in the premenopausal group [36]. Another study including women treated with L-thyroxine suppressive therapy for thyroid carcinoma found a decrease in bone resorption markers and an increase formation markers after short term recombinant human TSH stimulation in postmenopausal women but not in premenopausal women [37]. It was therefore important to exclude women taking hormone replacement therapy from our analysis. In the TRUST randomized controlled trial, after 1 year of levothyroxine daily substitution $(25-50 \mu \mathrm{g})$, there was no significant change on BMD and TBS in the 737 men and women included, and there was no difference to placebotreated participants [18]. The population was older than 65 years and had a subclinical hypothyroidism [18]. This interventional study suggests that even if there is an association between TSH and/or fT4 and BMD and/or TBS, a modest change in fT4 and/or TSH values does not modify BMD and/or TBS in the short term. This could mean that a long-term change is needed to highlight a clinical significance.
Influence of extreme ft4/TSH relationships on BMD or TBS in euthyroid postmenopausal women

This exploratory analysis was performed to highlight a possible difference between women close to subclinical hyperthyroidism or sub-clinical hypothyroidism values. For this analysis, we assumed that TSH and fT4 have an independent and inverse effect on BMD and TBS. Neither BMD values at the three measurement sites, nor TBS values differed between the two groups in the bivariate and multivariable analyses. The lack of difference observed may be due to the small sample size of the groups, or the fact that only TSH or only fT4 influences the BMD and TBS values. In a sample of 1426 peri-menopausal Dutch women, the BMD of participants with low TSH and high fT4 did not differ from that of other participants [29]. These results suggest that the interaction between thyroid hormones and the bone measurements in euthyroid subjects is small. It is still important to analyze these results with caution due to the potential bias from the categorization, and the unknown length of time of being in one or the other of the categories.

\section{Association between fT4 or TSH and incident osteoporotic fractures in euthyroid postmenopausal women}

We found that women in the lower normal TSH range had a higher incidence of major osteoporotic fracture 5 years later. Our results are consistent with the association found between TSH and TBS. In the OPUS 6-year prospective cohort study ( $n=1278)$, higher fT4 was associated with an increased risk of incident nonvertebral fracture, and higher TSH with a decrease of this risk [28]. In the OPENTHYRO register $(n=222,138$, mean follow-up 7.5 years), the risk of hip fractures increased with each SD decrease of TSH in both genders, while the risk of major osteoporotic fractures increased only in women (HR 1.32, 95\% CI 1.19-1.46) [38]. In a meta-analysis including 70,298 participants (mean age 64 years, $61.3 \%$ women), subclinical hyperthyroidism was associated with an increased risk of hip and other fractures, particularly among participants with TSH levels below $0.10 \mathrm{mU} / \mathrm{L}$ [13]. In another metaanalysis including 313,557 participants, subclinical hyperthyroidism was associated with a higher risk of all types of fractures [12]. Thus, there is a clear association in the literature between TSH and decreased risk of fracture [29]. However, caution should be used when interpreting the results because of the heterogeneity of the populations, and of the considered normal range for TSH values. 


\section{Strengths and limitations}

The main strength of this study is the design of the OsteoLaus and CoLaus cohorts. All blood tests and DXA exams were done under the same conditions. The same thyroid hormone normal values were used for all participants. Another strength is the purity of the studied sample reached through the exclusion criteria and confounders for osteoporosis. For example, we excluded the diabetic patients whose TBS is decreased while their BMD is paradoxically increased [39]. The limitations of the study are related to the relatively small sample size. In addition, due to the exclusion criteria, the results are not generalizable to all postmenopausal euthyroid women. We have not measured thyroid autoantibodies. Autoimmunity per se may affect bone structure. We have not measured fT3, which probably has a regulatory role. This could support measurement of fT3, fT4, and TSH in future studies, to better understand the link between thyroid hormones and bone. The effect of thyroid hormones on muscle has also been documented [38, 40]. This effect could alter the association between thyroid hormones and a marker of bone health. Inherent biases are the inter/intra individual or seasonal variation and the genetic polymorphism of the thyroid hormones. Minimizing those effects was possible thanks to the study size and the systematic collection of blood samples on the same time of the day [41]. TSH values were measured at baseline and not during the follow-up. This is a potential limitation of the study because the time of exposure to low and low-normal TSH may be an important factor influencing skeletal health [42].

\section{Conclusion}

This is the first study showing an association between TSH in the normal range and TBS, and with the fracture incidence after 5 years. Lower TSH may lead to a weaker bone structure and a higher fracture incidence without affecting the BMD. Even if there is a normal BMD, the TBS can already be degraded. The TBS seems then to be a more powerful tool to assess the fracture risk for secondary osteoporosis. It could help the clinician to assess smaller change in bone microarchitecture. Larger studies are needed to evaluate the influence of TSH on bone health.

Supplementary Information The online version contains supplementary material available at https://doi.org/10.1007/s00198-021-06081-4.

Acknowledgements The authors wish to thank all the participants of the study and the clinical teams who evaluated them.

Author contribution $\mathrm{CV}$ wrote most of the article; PMV collected data, made part of the statistical analysis, and wrote part of the article; OL revised the article for important intellectual content and wrote part of the article. PMV had full access to the data. OL is the guarantor of the study. GW, EGR, and DH participated in the development of the study and revised the article.

Funding Open access funding provided by University of Lausanne. The OsteoLaus study is supported by research grants from Lausanne University Hospital (Centre Hospitalier Universitaire Vaudois strategic plan funds) and the Swiss National Science Foundation (grant 32473B_156978). The CoLaus/PsyCoLaus study was and is supported by research grants from GlaxoSmithKline, the Faculty of Biology and Medicine of Lausanne, and the Swiss National Science Foundation (grants 3200B0-105993, 3200B0-118308, 33CSCO-122661, 33CS30139468 , 33CS30-148401, and 32473B-182210).

Data availability Some or all data generated or analyzed during this study are included in this published article or in the data repositories listed in References. The data presented in this study are available upon reasonable request to the corresponding author. The data are not publicly available due to the fact of confidentiality reasons.

Code availability Not applicable.

\section{Declarations}

Ethics approval and consent to participate The CoLaus and OsteoLaus studies were approved by the Institutional Ethics Committee of the University of Lausanne, which afterwards became the Ethics Commission of Canton Vaud (Commission cantonale d'éthique de la recherche sur l'être humain. Available from: http://www.cer-vd.ch). The studies were performed in agreement with the Helsinki Declaration and its former amendments, and in accordance with the applicable Swiss legislation. All participants gave their written informed consent before entering the study. Informed consent was obtained from all participants involved in the study.

Consent for publication Informed consent was obtained from all participants involved in the study.

Conflicts of interest Colin Vendrami, Pedro Marques-Vidal, Elena Gonzalez Rodriguez, Gérard Waeber, and Olivier Lamy declare that they have no conflict of interest related to this manuscript. Didier Hans is a co-owner of the TBS patent and holds stock options and royalties in the Medimaps group.

Additional declarations for articles in life science journals that report the results of studies involving humans and/or animals Not applicable.

Open Access This article is licensed under a Creative Commons Attribution-NonCommercial 4.0 International License, which permits any non-commercial use, sharing, adaptation, distribution and reproduction in any medium or format, as long as you give appropriate credit to the original author(s) and the source, provide a link to the Creative Commons licence, and indicate if changes were made. The images or other third party material in this article are included in the article's Creative Commons licence, unless indicated otherwise in a credit line to the material. If material is not included in the article's Creative Commons licence and your intended use is not permitted by statutory regulation or exceeds the permitted use, you will need to obtain permission directly from the copyright holder. To view a copy of this licence, visit http://creativecommons.org/licenses/by-nc/4.0/. 


\section{References}

1. Compston JE, McClung MR, Leslie WD (2019) Osteoporosis. The Lancet 393(10169):364-376. https://doi.org/10.1016/S01406736(18)32112-3

2. Miller PD, Siris ES, Barrett-Connor E et al (2002) Prediction of fracture risk in postmenopausal white women with peripheral bone densitometry: evidence from the National Osteoporosis Risk Assessment. J Bone Miner Res 17(12):2222-2230. https://doi.org/ 10.1359/jbmr.2002.17.12.2222

3. Svedbom A, Ivergård $\mathrm{M}$, Hernlund E, Rizzoli R, Kanis JA (2014) Epidemiology and economic burden of osteoporosis in Switzerland. Arch Osteoporos 9(1):187. https://doi.org/10.1007/ s11657-014-0187-y

4. Kanis JA, Burlet N, Cooper C et al (2008) European guidance for the diagnosis and management of osteoporosis in postmenopausal women. Osteoporos Int 19(4):399-428. https://doi.org/10.1007/ s00198-008-0560-z

5. University of Sheffield, UK (C) Centre for Metabolic Bone Diseases. Calculation Tool. FRAX ®Fracture Risk Assessment Tool. https://www.sheffield.ac.uk/FRAX/tool.aspx?lang=en

6. Silva BC, Leslie WD, Resch $\mathrm{H}$ et al (2014) Trabecular bone score: a noninvasive analytical method based upon the DXA image. J Bone Miner Res 29(3):518-530. https://doi.org/10.1002/jbmr. 2176

7. McCloskey EV, Odén A, Harvey NC et al (2016) A meta-analysis of trabecular bone score in fracture risk prediction and its relationship to FRAX: TBS in fracture risk prediction and relationship to FRAX. J Bone Miner Res 31(5):940-948. https://doi.org/10.1002/ jbmr.2734

8. Ripamonti C, Lisi L, Buffa A, Gnudi S, Caudarella R (2018) The trabecular bone score predicts spine fragility fractures in postmenopausal Caucasian women without osteoporosis independently of bone mineral density. Med Arch 72(1):46-50. https://doi.org/10. 5455/medarh.2018.72.46-50

9. Padlina I, Gonzalez-Rodriguez E, Hans D et al (2017) The lumbar spine age-related degenerative disease influences the BMD not the TBS: the Osteolaus cohort. Osteoporos Int 28(3):909-915. https:// doi.org/10.1007/s00198-016-3829-7

10. Hollowell JG, Staehling NW, Flanders WD et al (2002) Serum TSH, T4, and thyroid antibodies in the United States population (1988 to 1994): National Health and Nutrition Examination Survey (NHANES III). J Clin Endocrinol Metab 87(2):489-499. https://doi.org/10.1210/jcem.87.2.8182

11. Cooper DS, Biondi B (2012) Subclinical thyroid disease. The Lancet 379(9821):1142-1154. https://doi.org/10.1016/S01406736(11)60276-6

12. Zhu H, Zhang J, Wang J, Zhao X, Gu M (2020) Association of subclinical thyroid dysfunction with bone mineral density and fracture: a meta-analysis of prospective cohort studies. Endocrine 67(3):685-698. https://doi.org/10.1007/s12020-019-02110-9

13. Blum MR, Bauer DC, Collet T-H et al (2015) Subclinical thyroid dysfunction and fracture risk: a meta-analysis. JAMA 313(20):2055-2065. https://doi.org/10.1001/jama.2015.5161

14. Segna D, Bauer DC, Feller M et al (2018) Association between subclinical thyroid dysfunction and change in bone mineral density in prospective cohorts. J Intern Med 283(1):56-72. https:// doi.org/10.1111/joim.12688

15. Aubert CE, Floriani C, Bauer DC et al (2017) Thyroid function tests in the reference range and fracture: individual participant analysis of prospective cohorts. J Clin Endocrinol Metab 102(8):2719-2728. https://doi.org/10.1210/jc.2017-00294

16. Hans D, Šteňová E, Lamy O (2017) The trabecular bone score (TBS) complements DXA and the FRAX as a fracture risk assessment tool in routine clinical practice. Curr Osteoporos Rep 15(6):521-531. https://doi.org/10.1007/s11914-017-0410-z

17. Hwangbo Y, Kim JH, Kim SW et al (2016) High-normal free thyroxine levels are associated with low trabecular bone scores in euthyroid postmenopausal women. Osteoporos Int 27(2):457-462. https://doi.org/10.1007/s00198-015-3270-3

18. Gonzalez Rodriguez E, Stuber M, Del Giovane C et al (2019) Skeletal effects of levothyroxine for subclinical hypothyroidism in older adults: a TRUST Randomized Trial Nested Study. J Clin Endocrinol Metab 105(1):336-343. https://doi.org/10.1210/clinem/dgz058

19. Firmann M, Mayor V, Vidal PM et al (2008) The CoLaus study: a population-based study to investigate the epidemiology and genetic determinants of cardiovascular risk factors and metabolic syndrome. BMC Cardiovasc Disord 8:6. https://doi.org/10.1186/ 1471-2261-8-6

20. Marques-Vidal P, Bochud M, Bastardot $F$ et al (n.d.) Assessing the associations between mental disorders, cardiovascular risk factors, and cardiovascular disease : the CoLaus/PsyCoLaus study.

21. Shevroja E, Marques-Vidal P, Aubry-Rozier B et al (2019) Cohort profile: the OsteoLaus study. Int J Epidemiol 48(4):1046-1047. https://doi.org/10.1093/ije/dyy276

22. Genant HK, Wu CY, van Kuijk C, Nevitt MC (1993) Vertebral fracture assessment using a semiquantitative technique. J Bone Miner Res 8(9):1137-1148. https://doi.org/10.1002/jbmr.56500 80915

23. Skeletal sites for osteoporosis diagnosis: the 2005 ISCD Official Positions-ScienceDirect. Accessed March 4, 2019. https://www. sciencedirect.com/science/article/pii/S1094695006001855?via\% 3Dihub

24. Shevroja E, Aubry-Rozier B, Hans G et al (2019) Clinical Performance of the Updated Trabecular Bone Score (TBS) Algorithm, Which Accounts for the Soft Tissue Thickness: The OsteoLaus Study. J Bone Miner Res 34(12):2229-2237. https://doi.org/10. 1002/jbmr.3851

25. Roche Diagnostics GmbH (2009) Reference Intervals for children and adults, Elecsys and Cobas e Analyzers, Elecsys Thyroid Tests

26. Moon JH, Kim KM, Oh TJ et al (2017) The effect of TSH suppression on vertebral trabecular bone scores in patients with differentiated thyroid carcinoma. J Clin Endocrinol Metab 102(1):78-85. https://doi.org/10.1210/jc.2016-2740

27. De Mingo Dominguez ML, Guadalix Iglesias S, Martin-Arriscado Arroba C et al (2018) Low trabecular bone score in postmenopausal women with differentiated thyroid carcinoma after long-term TSH suppressive therapy. Endocrine 62(1):166-173. https://doi. org/10.1007/s12020-018-1671-8

28. Mazziotti G, Formenti AM, Frara S, Olivetti R, Banfi G, Memo $M$ et al (2018) High prevalence of radiological vertebral fractures in women on thyroid-stimulating hormone-suppressive therapy for thyroid carcinoma. J Clin Endocrinol Metab 103(3):956-964. https://doi.org/10.1210/jc.2017-01986

29. Mazziotti G, Porcelli T, Patelli I, Vescovi PP, Giustina A (2010) Serum TSH values and risk of vertebral fractures in euthyroid post-menopausal women with low bone mineral density. Bone 46(3):747-751. https://doi.org/10.1016/j.bone.2009.10.031

30. Murphy E, Glüer CC, Reid DM et al (2010) Thyroid function within the upper normal range is associated with reduced bone mineral density and an increased risk of nonvertebral fractures in healthy euthyroid postmenopausal women. J Clin Endocrinol Metab 95(7):3173-3181. https://doi.org/10.1210/jc.2009-2630

31. van Rijn LE, Pop VJ, Williams GR (2014) Low bone mineral density is related to high physiological levels of free thyroxine in peri-menopausal women. Eur J Endocrinol 170(3):461-468. https://doi.org/10.1530/EJE-13-0769 
32. Abe E, Marians RC, Yu W et al (2003) TSH is a negative regulator of skeletal remodeling. Cell 115(2):151-162. https://doi.org/10. 1016/S0092-8674(03)00771-2

33. Sun L, Zhu L-L, Lu P et al (2013) Genetic confirmation for a central role for $\mathrm{TNF} \alpha$ in the direct action of thyroid stimulating hormone on the skeleton. Proc Natl Acad Sci 110(24):9891-9896. https://doi.org/10.1073/pnas.1308336110

34. Ma R, Morshed S, Latif R, Zaidi M, Davies TF (2011) The influence of thyroid-stimulating hormone and thyroid-stimulating hormone receptor antibodies on osteoclastogenesis. Thyroid Off J Am Thyroid Assoc 21(8):897-906. https://doi.org/10.1089/thy.2010. 0457

35. Bassett JHD, Williams AJ, Murphy E et al (2008) A lack of thyroid hormones rather than excess thyrotropin causes abnormal skeletal development in hypothyroidism. Mol Endocrinol Baltim Md 22(2):501-512. https://doi.org/10.1210/me.2007-0221

36. Martini G, Gennari L, De Paola V et al (2008) The effects of recombinant TSH on bone turnover markers and serum osteoprotegerin and RANKL levels. Thyroid 18(4):455-460. https://doi. org/10.1089/thy.2007.0166

37. Mazziotti G, Sorvillo F, Piscopo M et al (2005) Recombinant human TSH modulates in vivo C-telopeptides of type-1 collagen and bone alkaline phosphatase, but not osteoprotegerin production in postmenopausal women monitored for differentiated thyroid carcinoma. J Bone Miner Res Off J Am Soc Bone Miner Res 20(3):480-486. https://doi.org/10.1359/JBMR.041126
38. Bloise FF, Cordeiro A, Ortiga-Carvalho TM (2018) Role of thyroid hormone in skeletal muscle physiology. J Endocrinol 236(1):R57-R68. https://doi.org/10.1530/JOE-16-0611

39 Leslie WD, Aubry-Rozier B, Lamy O, Hans D, for the Manitoba Bone Density Program (2013) TBS (trabecular bone score) and diabetes-related fracture risk. J Clin Endocrinol Metab. 98(2):602-9. https://doi.org/10.1210/jc.2012-3118

40. Salvatore D, Simonides WS, Dentice M, Zavacki AM, Larsen PR (2014) Thyroid hormones and skeletal muscle new insights and potential implications. Nat Rev Endocrinol 10(4):206-214. https:// doi.org/10.1038/nrendo.2013.238

41. Strich D, Karavani G, Levin S, Edri S, Gillis D (2016) Normal limits for serum thyrotropin vary greatly depending on method. Clin Endocrinol (Oxf) 85(1):110-115. https://doi.org/10.1111/ cen. 12970

42. Abrahamsen B, Jørgensen HL, Laulund AS, Nybo M, Brix TH, Hegedüs L (2014) Low serum thyrotropin level and duration of suppression as a predictor of major osteoporotic fractures-the OPENTHYRO register cohort. J Bone Miner Res 29(9):20402050. https://doi.org/10.1002/jbmr.2244

Publisher's note Springer Nature remains neutral with regard to jurisdictional claims in published maps and institutional affiliations. 